\title{
ÁlCOOL E DROGAS NA ATENÇÃO PRIMÁRIA: AVALIANDO ESTRATÉGIAS DE CAPACITAÇÃO'
}

Isabel Cristina Weiss de Souza*

Telmo Mota Ronzani

\begin{abstract}
RESUMO. O presente estudo teve por objetivo analisar práticas de prevenção ao uso de risco de álcool e outras drogas adotadas por enfermeiros da Atenção Primária à Saúde (APS) capacitados nas modalidades presencial e à distância e por enfermeiros não capacitados. Os dados foram coletados através de roteiro de entrevista semiestruturada com sete enfermeiras que atuam na APS, e o procedimento analítico adotado foi a análise de conteúdo. Procedeu-se à análise de seis temas, a saber: capacitação; práticas; atitudes; crenças sobre álcool e outras drogas; conhecimentos específicos sobre tratamentos para álcool e outras drogas; sentimentos envolvidos ao lidar com o tema álcool e outras drogas. Os resultados sugerem que ainda prevalece a subutilização de práticas baseadas em evidências no tocante a álcool e outras drogas, e que um espaço de discussão permanente subsequente às capacitações serviria de reforço, em médio prazo, para a capacitação, aumentando o senso de autoeficácia para lidar com esta demanda.
\end{abstract}

Palavras-chave: Capacitação em álcool e outras drogas; atenção primária à saúde; práticas de prevenção.

\section{ALCOHOL AND DRUGS IN PRIMARY CARE: EVALUATING STRATEGIES FOR TRAINING}

\begin{abstract}
This study aims to examine practices of prevention against the risky use of alcohol and other drugs adopted amongst Primary Health Care (PHC) nurses trained in different modalities - present and at a distance and also un-trained professionals. Data were collected following a semi-structured model of interview with seven nurses who worked in the PHC. A content analysis was adopted as the analytical procedure. Six themes were analyzed: training, practices, attitudes, beliefs about alcohol and other drugs; specific knowledge of treatments for alcohol and other drugs; feelings involved in dealing with the topics of alcohol and other drugs. The results suggest that the underutilization of evidence-based practice in relation to alcohol and other drugs is still prevailing, and that a permanent forum for discussion would serve to strengthen, at medium term, for training, increasing the sense of self-efficacy for dealing with this demand.
\end{abstract}

Key words: Training in alcohol and other drugs; primary health care; prevention practices.

\section{ALCOHOL Y DROGAS EN LA ATENCIÓN PRIMARIA: EVALUANDO ESTRATEGIAS DE CAPACITACIÓN}

\begin{abstract}
RESUMEN. El presente estudio tiene por objetivo analizar prácticas de prevención al uso de riesgo del alcohol y otras drogas adoptadas entre enfermeros de la Atención Primaria a la Salud (APS) capacitados en modalidades presencial, a la distancia y profesionales no capacitados. Los datos fueron colectados a través del itinerario de la entrevista semiestructurada con siete enfermeras que actúan en la APS, siendo el análisis de contenido y procedimiento analítico adoptado. Se procedió al análisis de seis temas: capacitación; prácticas; actitudes; creencias sobre alcohol y otras drogas; conocimientos específicos sobre tratamientos para alcohol y otras drogas; sentimientos involucrados al lidiar con el tema del alcohol y otras drogas. Los resultados sugieren que todavía prevalezca la subutilización de prácticas fundamentadas en evidencia en lo tocante al alcohol y otras drogas, y que un espacio de discusión permanente subsecuente a las capacitaciones serviría de refuerzo, a medio plazo, para la capacitación, aumentando el sentido de autoeficacia para lidiar con ésta demanda.
\end{abstract}

Palabras-clave: Capacitación en alcohol y otras drogas; atención primaria a la salud; prácticas de prevención.

Apoio: CNPq; FAPEMIG.

Psicóloga,terapeuta cognitivo-comportamental, mestre em Saúde Coletiva pela Faculdade de Medicina da Universidade Federal de Juiz de Fora (UFJF), pesquisadora do Pólo de Pesquisa em Psicologia Social e Saúde Coletiva da UFJF.

\# Professor Adjunto da Universidade Federal de Juiz de Fora (UFJF), Pós-Doutorado pela Universidade de São Paulo (USP) e estágio pós-doutoral pela University of Connecticut Health Center (UCHC). Bolsista de Produtivdade CNPq e Pesquisador Mineiro FAPEMIG. Coordenador do Pólo de Pesquisa em Psicologia Social e Saúde Coletiva da UFJF. 
Nos anos recentes o consumo de álcool e de outras drogas tem aumentado em todo o mundo, em magnitude suficiente para justificar uma abordagem de saúde pública para intervenções precoces (WHO Assist Working Group, 2002). Além disso, por ser este um dos mais graves problemas de saúde pública no mundo, torna-se necessária a construção de políticas públicas eficazes e capazes de enfrentar com sucesso esta problemática (Ministério da Saúde, 2004).

Dados apresentados no I Levantamento Nacional sobre os Padrões de Consumo de Álcool na População Brasileira (Laranjeira, Pinsky, Zaleski, \& Caetano, 2007) confirmam que, do total da população brasileira com 18 anos ou mais, 3\% fazem uso nocivo (padrão de uso que já está causando dano físico ou mental à saúde e muitas vezes associado a vários tipos de consequências sociais adversas, segundo o CID -10) e $9 \%$ são dependentes de bebidas alcoólicas, somando um total de $12 \%$ da população brasileira com problemas com o álcool, índice muito significativo em termos de saúde pública.

Os danos relacionados ao consumo extrapolam as mortes e doenças e se estendem à violência doméstica, lesões corporais, tentativas de homicídio, homicídios consumados, conflitos interpessoais, acidentes com automotores (em geral homens jovens de até 25 anos de idade, longe do estereótipo do dependente crônico, segundo pesquisas em países desenvolvidos) e intoxicações (Laranjeira et al., 2007).

Com recursos públicos e tempo limitados para serem aplicados na Atenção Primária à Saúde (APS) diante de tantos problemas que podem e devem ser prevenidos, torna-se fundamental tomar medidas de prevenção que apresentem comprovação de boa relação custo-benefício.

Com a finalidade de elaborar um ranking de prioridades em prevenção, foi criada nos Estados Unidos uma comissão denominada National Commission on Prevention Priorities, com fundos do Centers for Disease Control and Prevention (CDC) e da Agency for Health Care Research and Quality (AHRQ), a qual identificou, em 2006, as práticas de rastreamento de abuso de álcool seguidas de aconselhamento na APS como uma entre as cinco mais efetivas e custoefetivas, à frente de outros vinte serviços também efetivos (Solberg, Maciosek, \& Edwards, 2008).

A APS é a instância em que se pode responder de maneira mais oportuna às necessidades de saúde da população, pois é nela que os profissionais têm contato com grande número de pessoas que deveriam ser abordadas em relação à forma como usam álcool e outras drogas, devido ao fato de já apresentarem algum tipo de problema relacionado ao consumo. Isto pode evitar que muitos deles evoluam para um quadro de dependência da substância ou problemas associados ao uso da substância (Ministério da Saúde, 2004; Ronzani, 2005).

Diante disso, o uso problemático de álcool foi escolhido pelo Ministério da Saúde como componente da lista dos dez problemas de saúde a serem priorizados pelo Programa de Saúde da Família (PSF), em função do reconhecimento do problema como muito expressivo em termos de saúde pública, mas principalmente numa tentativa de incentivar práticas de detecção precoce, prevenindo os danos que causa o uso do álcool (Ronzani, 2005).

Neste sentido, o Ministério da Saúde publicou, em 3 de maio de 2002, a Portaria GM / MS n. 816, de 30 de abril de 2002, e a Portaria SAS / MS n. 305, de 30 de abril de 2002, as quais estabeleceram diretrizes para a política de álcool e outras drogas, propondo um conjunto de ações sistemáticas relativas ao tratamento e prevenção no campo de álcool e outras drogas.

A Portaria GM/MS n. 816, de 30 de abril de 2002, já destacava a necessidade de ampliar a oferta de atendimento a essa clientela na rede do SUS e de reformular e adequar o modelo de assistência oferecido pelo SUS ao usuário de álcool e outras drogas, aperfeiçoando-o e qualificando-o. Para isto várias ações estão previstas, entre elas a de promover, em articulação com instituições formadoras, a capacitação e supervisão das equipes de Atenção Básica, serviços e programas locais de saúde mental.

É muito relevante para os profissionais do SUS pensar e discutir políticas e estratégias de capacitação para trabalhar com o problema de consumo de álcool e outras drogas. A Organização Mundial de Saúde (OMS) apoiou o desenvolvimento de instrumentos de triagem que são ferramentas importantes para identificar qual o nível de uso, ajudando o profissional de saúde a definir a estratégia de intervenção mais adequada (Humeniuk \& Poznyak, 2004).

Um dos instrumentos desenvolvidos é o Alcohol Use Disorders Identification Test - AUDIT, citado por Saunders, Aasland, Babor, de la Fuente e Grant (1993), usado para detectar padrões de uso excessivo de álcool e problemas associados, o qual tem se mostrado compatível com a APS e muito útil para intervenções preventivas. Este instrumento foi validado para países em desenvolvimento e vem demonstrando um desempenho de igual ou melhor precisão em comparação com outros testes de rastreamento (Babor \& Higgins-Biddle, 2001). 
Com a detecção do padrão de consumo de álcool por meio da aplicação do AUDIT, pode ser realizada uma intervenção adequada para cada nível de uso da substância. A "Intervenção Breve" (IB) é uma abordagem relacionada à prevenção primária ou secundária para usuários de álcool e outras drogas que tem como foco a mudança de comportamento do paciente através de um atendimento com tempo limitado e que pode ser realizado por profissionais de diferentes formações.

O objetivo da IB na Atenção Primária é promover a percepção do paciente de que o uso da substância está colocando-o em risco e encorajá-lo a reduzir ou deixar o uso a partir de estratégias e metas construídas entre o profissional e o paciente. Existem evidências substanciais dos benefícios da triagem e intervenção breve para o problema do uso de álcool nos serviços de Atenção Primária à Saúde (Babor, Higgins-Biddle, Dauser, Higgins, \& Burleson, 2005; Babor et al., 2007; Kaner et al., 2009).

Pesquisas apontam que intervenções breves também podem ser efetivas neste contexto para outras drogas que não o álcool, pois evidências sugerem que essas intervenções podem funcionar para maconha, benzodiazepínicos, anfetaminas, opioides e cocaína (Humeniuk \& Poznyak, 2004).

A formação do profissional de saúde ainda é voltada para práticas de reabilitação e é bastante deficitária quanto ao uso de substâncias psicoativas, tanto nos países desenvolvidos quanto naqueles em desenvolvimento (Luis et al., 2004).

Estudos confirmam que o mais importante aspecto de um programa educacional em saúde é conseguir disponibilizar o aprendizado da prática clínica para um cuidado que vá muito além do conhecimento técnico e de mudança de atitude (Cantillon \& Jones, 1999; Rassool \& Rawaf, 2008). Os profissionais acabam por eleger como prioridades de rastreamento em sua prática diária na APS problemas de saúde sobre os quais eles detêm conhecimento e ferramentas comprovadamente eficazes para atuarem (Cabana et al., 1999).

A inserção da prática de prevenção contra álcool e outras drogas na rotina da assistência estaria condicionada, dentro desta perspectiva, ao treinamento dos profissionais na prática clínica para, por exemplo, identificar os casos precocemente e saber como proceder quando estes são confirmados. Como alternativa na educação permanente em saúde a Educação à Distância $(\mathrm{EaD})$ pode favorecer a interdisciplinaridade nas equipes, a interação entre atores e a otimização dos processos de comunicação e informação (Garcia \& Baptista, 2007), além de propiciar a aprendizagem contínua e a socialização de conhecimentos (Oliveira, 2007), e como proposta não compulsória de ensino, estimular a autogestão, autoformação e autoavaliação, condições fundamentais para uma aprendizagem que possa se sustentar a longo prazo. No entanto, geralmente as capacitações partem da iniciativa do Governo ou de pesquisadores, muitas vezes na modalidade presencial, com atuação pontual e sem supervisão das ações, o que dificulta a incorporação de mudanças na prática de saúde (Camargo Júnior et al., 2008).

Neste contexto, evidencia-se a necessidade de capacitar o profissional da saúde para atuar na prevenção ao uso abusivo de álcool e outras drogas, assim como avaliar o que vem sendo feito, uma vez que muitas vezes recursos públicos são investidos para mudar o cenário de negligência diante desta demanda e expectativas são criadas neste sentido.

Este estudo tem como objetivo avaliar as práticas de prevenção ao uso abusivo de álcool e outras drogas adotadas em dois municípios da Zona da Mata Mineira (Brasil) nos quais os profissionais da Saúde receberam capacitação para atuar nesta perspectiva em comparação com as práticas desenvolvidas em um terceiro município da mesma região que não promoveu essa capacitação.

\section{MÉTODO}

\section{Participantes}

O estudo se caracteriza como de caráter exploratório do tipo descritivo e analítico, com amostragem intencional e definição a priori dos participantes da pesquisa a partir comparação das modalidades de capacitação adotadas.

$\mathrm{O}$ estudo foi realizado em três municípios da Zona da Mata de Minas Gerais. Por questões éticas, optouse por denominá-los Município A, Município B e Município C.

As participantes do município A receberam capacitação de 90 horas na modalidade à distância, promovida pela Senad (Secretaria Nacional de Políticas sobre Drogas) em parceria com o Ministério da Saúde e diversas universidades, sob coordenação da Universidade Federal de São Paulo (Unifesp), entre os meses de dezembro de 2006 e fevereiro de 2007.

O objetivo do curso, denominado Supera (Sistema para Detecção do Uso Abusivo e Dependência de Substâncias Psicoativas: Encaminhamento, Intervenção Breve, Reinserção Social e Acompanhamento), como o próprio nome o diz, é capacitar profissionais que atuam na saúde para uso de instrumentos de detecção do uso abusivo de álcool e outras substâncias e para realização da IB. 
A Senad forneceu uma listagem com os 52 nomes e respectivos contatos dos alunos do Supera do município A, onde seria realizada parte desta pesquisa. Optou-se por delimitar o estudo à APS devido à posição sabidamente estratégica que esta ocupa no contexto da prevenção de doenças e promoção de saúde.

Dentre os 52 nomes, apenas vinte eram de profissionais da APS do município A, e destes, apenas dois nomes preencheram o critério de inclusão na pesquisa: ter sido capacitado exclusivamente na modalidade à distância na área de álcool e outras drogas. A definição destas duas participantes mulheres e enfermeiras - determinou o gênero e a categoria profissional para os outros dois grupos.

As participantes do município B compõem equipes do PSF que foram capacitadas presencialmente por um grupo de pesquisadores da Universidade Federal de Juiz de Fora (UFJF) como parte integrante de um projeto denominado "Disseminação de Práticas de Prevenção ao Uso de Risco de Álcool em Serviços de APS da Zona da Mata Mineira". O objetivo desse projeto, que foi financiado pelo CNPq (Edital MCT/CNPq/MS-SCTIE-DECT 23/2006 - Estudo de Gestão Em Saúde) consistiu em implementar estratégias de prevenção ao uso de risco de álcool na rotina de serviços de APS de municípios de pequeno porte (menos de 100.000 habitantes).

$\mathrm{O}$ estudo passou por várias etapas compreendendo contatos com os gestores do município, capacitação presencial de oito horas envolvendo 113 profissionais ao todo e acompanhamento por seis meses após a realização do treinamento -, e foi concluído em junho de 2007 (Ronzani, Mota, \& Souza, 2009).

$\mathrm{O}$ município $\mathrm{C}$ foi escolhido pelo fato de os profissionais da APS nunca terem se submetido à capacitação na área de álcool e outras drogas e por situar-se muito próximo dos municípios A e B e ter características muito semelhantes às do município $\mathrm{B}$ em termos de número de habitantes, recursos de ensino disponíveis, hábitos e costumes.

Destarte, foram formados três grupos: o grupo treinado presencialmente, no município B (GT), o grupo capacitado pelo Supera (GS) e grupo não treinado (GNT). As duas participantes do grupo GT foram escolhidas por sorteio entre as enfermeiras das três equipes de PSF credenciadas no município B. O grupo GNT foi composto por duas enfermeiras sorteadas entre UBSs do município $\mathrm{C}$ e uma enfermeira do município A que nunca foram submetidas à capacitação na área.
A amostra então foi composta por sete enfermeiras, das quais cinco se situam na faixa etária entre 26 e 31 anos (entre 3 e 6 anos de formadas), uma tem 35 anos (11 anos de formada) e uma tem 50 anos (25 anos de formada).

Apenas uma enfermeira do município A pôde compor o GNT (GNT3), pois somente ela declarou não ter passado por nenhuma capacitação em álcool, outras drogas e tabaco. Sua inclusão no estudo se deu como opção para, no caso de encontrarmos diferenças importantes na atuação de GNT, podermos pensá-las quanto à falta de treinamento, e não somente pelo fato de tratar-se de uma população que tem acesso restrito à informação, pois o município $\mathrm{C}$ não conta com universidades, centros de formação e nem mesmo tem vida cultural ativa, diferentemente do município A.

O município A tem aproximadamente 500.000 habitantes e oitenta e duas equipes de PSF; o município B tem aproximadamente 9.750 habitantes e três equipes de PSF, e o município C, 8.910 habitantes e três equipes de PSF. Todas as entrevistadas são integrantes de equipes de PSF em seus municípios.

As profissionais capacitadas haviam recebido capacitação por iniciativas isoladas ou de grupo de pesquisadores (GT1 e GT2), ou por iniciativa própria (GS1 e GS2), e não propriamente como parte de uma política local que reconhecesse sua importância ou que buscasse reorganizar saberes e conhecimentos a partir das necessidades de ordem prática.

\section{Instrumentos}

Os instrumentos de pesquisa, os quais foram adaptados do estudo de Ronzani (2005) e dirigidos a todas as profissionais de APS participantes do estudo, compreenderam:

1. Questionário sociodemográfico, que identifica as principais características dos profissionais entrevistados: sexo, idade, formação profissional (ensino fundamental, médio, superior, pósgraduado), tempo de formado, instituição onde se graduou (tendo se graduado no ensino superior), estado civil, tempo de ingresso no PSF, critério de ingresso (concurso ou contrato);

2. Roteiro de entrevista semiestruturada sobre práticas de prevenção e concepções acerca dos problemas relacionados ao uso de álcool e outras drogas, aplicado entre os profissionais dos grupos GT, GS e GNT, testado em piloto realizado junto a outros profissionais de saúde do município A, em outubro de 2008. 


\section{Delineamento da pesquisa}

Utilizou-se para a coleta de dados a entrevista semiestruturada (Minayo, 2006), combinando perguntas fechadas e abertas a fim de que as entrevistadas pudessem discorrer com mais liberdade sobre os temas pesquisados.

A entrevista viabilizou acesso às informações de que os três grupos dispõem sobre práticas de prevenção ao uso abusivo de álcool e outras drogas, assim como às suas crenças e atitudes em relação ao usuário.

Este projeto foi aprovado pelo Comitê de Ética em Pesquisa da UFJF, assim como pelas secretarias de saúde dos municípios envolvidos (GT, GS e GNT).

As entrevistas com as sete profissionais envolvidas foram feitas durante o mês de novembro de 2008. As profissionais entrevistadas assinaram um termo formal de consentimento. Foram coletados dados sociodemográficos e em seguida deu-se início às entrevistas.

As profissionais que compõem o GS e o GT concluíram suas capacitações até junho de 2007, o que sugere haver um lapso de tempo conveniente para se proceder à avaliação da assimilação dos conteúdos em suas práticas e crenças.

Foram analisados neste estudo dados provenientes da metodologia qualitativa, através da análise de conteúdo do tipo estrutural e temático (Bardin, 1977). Após a transcrição dos relatos, recortamo-los em unidades comparáveis de categorização para daí procedermos à análise dos seis temas: Capacitação; Práticas; Atitudes; Crenças sobre álcool e outras drogas; Conhecimentos específicos sobre tratamentos para álcool e outras drogas; Sentimentos envolvidos ao lidar com o tema álcool e outras drogas e seus respectivos subtemas.

$\mathrm{Na}$ realização da análise vertical das entrevistas de cada grupo, buscamos identificar os elementos mais significativos das falas e os agrupamos em categorias e subcategorias. Em seguida realizamos a categorização horizontal dos relatos apresentados na entrevista dos três grupos (GT, GS e GNT), e por meio da análise da frequência avaliamos a tendência geral dos participantes em relação a cada tema estudado.

Ao analisarmos as atitudes das profissionais entrevistadas, certamente nos valeu atentar para seus sentimentos envolvidos, pois as atitudes que orientam a relação entre o indivíduo e o ambiente apresentam um componente cognitivo (analisado aqui através das crenças), um afetivo/emotivo e um comportamental (analisado, neste nosso estudo, por meio das práticas de prevenção em álcool e outras drogas adotadas) (Cavazza, 2008).

\section{RESULTADOS E DISCUSSÃO}

O ingresso das profissionais na APS foi imediato à graduação para cinco das sete entrevistadas, e para duas delas o ingresso ocorreu dois anos após a conclusão do curso. Quatro delas têm vínculo empregatício com o Programa de Saúde da Família (PSF) por concurso público, e três, por contrato temporário.

Cinco delas graduaram-se em universidade pública e duas, em universidade particular. Todas as entrevistadas têm curso de pós-graduação.

Quanto ao fato de escolherem trabalhar na APS/PSF, seis responderam terem-no feito por identificar-se com o trabalho e uma afirmou ser a oportunidade de trabalho mais acessível.

No tocante à satisfação no trabalho, numa escala entre nada satisfeito e totalmente satisfeito, quatro se dizem satisfeitas e três, totalmente satisfeitas.

Seis das participantes declararam realizar sempre atividades de prevenção e promoção e uma relatou realizá-las quase sempre.

Entre as respostas relativas à motivação para trabalhos de prevenção ao uso nocivo de álcool, numa escala entre totalmente desmotivado e totalmente motivado, uma se diz totalmente motivada, cinco se dizem motivadas, uma se diz neutra.

$\mathrm{Na}$ realidade, a análise das entrevistas denota que o tema álcool e drogas parece negligenciado no contexto de atuação destas profissionais, principalmente no tocante às ações de promoção de saúde, com amplo reconhecimento do despreparo para lidar com ele.

A análise horizontal demonstrou uma grande homogeneidade entre os grupos e pouquíssima divergência nas respostas.

\section{Tema 1: Capacitação}

Das sete entrevistadas, somente uma afirmou não ter tido nenhum contato formal, por mínimo que fosse, com o tema álcool e outras drogas, apesar de seus nove anos de prática em APS e de atuar em município onde há um CAPS AD, o qual é responsável por desenvolver projetos terapêuticos e comunitários e assessorar e dar cobertura ao trabalho na APS, oferecendo o chamado Apoio Matricial (Ministério da Saúde, 2004). 


\begin{abstract}
"Olha, eu acho assim que são muito insuficientes, né? Porque, por exemplo, nesses nove anos que eu estou aqui na UBS praticamente o que a gente teve foi com relação ao CAPS AD, mas...a nível de fluxograma, de como é organizado o serviço (...) Algo específico pra álcool e drogas,eu não" (GNT3).
\end{abstract}

A política de álcool e outras drogas em nosso país, principalmente no tocante à capacitação, talvez pela pouca familiaridade com as prioridades da política do álcool, parece ainda ausente da agenda da APS, apesar de presente entre as dez prioridades na APS e descrita como uma política muito bem articulada que prevê a formação de recursos humanos na área (Laranjeira \& Romano, 2004), como podemos constatar na fala de uma das entrevistadas (GT2):

\begin{abstract}
"Acho que todo profissional que trabalha na APS devia passar por essa capacitação, devia ser assim, uma coisa... é... sinceramente, porque eu não sei se você conhece a Portaria 648, que trata da APS? Ela fala de hanseníase, de tuberculose, da saúde da criança... ela não fala nada de álcool e drogas. Não tem nenhum indicador nosso que fale de álcool e drogas. (...) Acredito que se houvesse (...) uma meta em torno disso aí... no combate ao álcool e drogas". (GT2)
\end{abstract}

$\mathrm{Na}$ prática, o processo de incorporação da informação é complexo e neste sentido é vasta na literatura a indicação de formação permanente de recursos humanos (Bosch-Capblanch \& Garner, 2008; Camargo Júnior et al., 2008; Garcia \& Batista, 2007). Muito já se esperou que a formação acadêmica fosse suficiente, mas sabe-se que prescrição de trabalho não se traduz em trabalho realizado. O que se espera é a superação do modelo de serviços centrados em procedimentos e a implantação de serviços em que se permitam a discussão, análise, supervisão e reflexão da prática no cotidiano do trabalho com base na integralidade e autonomia (Ceccim, 2007).

\footnotetext{
"Acho que quando retoma um pouco essa coisa do álcool, da droga, a gente volta... vai voltando, né? (...) Mas acho que ele vai se perdendo um pouco, depois se não tiver uma continuidade". (GS1)
}

“(...) Tem que ter desfecho e esse desfecho, pelo menos eu percebi que se dá na discussão, porque na discussão eles encontram o quê? Soluções, a troca de experiência, a troca de ideias... na minha área tem um mesmo caso, eu fiz assim, deu certo
(...) Eu acredito que não foi para frente porque a gente deixou de ter esse acompanhamento, esse acompanhamento era muito bom!" (GT2)

Todas as integrantes dos grupos treinados, presencialmente ou à distância, referiram-se, com ênfase, à necessidade de continuarem as ações educativas às quais foram submetidas. Ressaltaram a falta de contato humano, de troca de experiências e de adequação do conteúdo às realidades cotidianas do cuidado, reafirmando o que a literatura já consagrou como essencial no processo de educação na saúde: onde a política de formação de recursos humanos é permanente, a incorporação de informação é mais eficaz.

\section{Tema 2: Práticas}

As quatro profissionais que receberam capacitação no último ano declararam não ter dado continuidade às ações treinadas. No grupo GS, uma das entrevistadas declarou que houve influência positiva em sua prática individual diária, mas, pelo fato de em sua equipe de trabalho somente ela ter sido treinada, não houve incorporação na rotina. Por sua vez, o grupo GT declarou ter abandonado as práticas após o término do acompanhamento das tutoras:

"Nós fizemos a capacitação em abril, foi até
quando a gente teve... eu acredito que
durante seis meses elas ainda vieram aqui nos
acompanhando (...) Depois disso, durante uns
dois ou três meses a gente ainda seguiu
sozinho, depois a gente murchou...aí paramos
com as discussões". (GT2)

Ficou evidente nas respostas que nenhuma das entrevistadas realiza avaliação de padrão de consumo de álcool e outras drogas utilizando-se de instrumentos validados para este fim: (...) "Vamos naquele que a gente sabe que bebe. Paciente caído, na rua... acho que isso aí já é uma forma de eu avaliar (...) Informalmente". (GT2). "Prejudica, às vezes, o familiar, o vizinho... aí a gente faz uma intervenção". (GS1).

Entre as entrevistadas, o enfoque da intervenção em álcool e outras drogas ainda é curativo, com ênfase na doença, ficando de lado a vertente preventiva e a atuação sanitária, em contraposição ao princípio da integralidade, pressuposto básico do Programa Saúde da Família, que visa a uma apreensão mais abrangente das necessidades de saúde do sujeito (Alves, 2005). Esse procedimento está igualmente em contraposição ao que foi proposto nas capacitações a que foram 
submetidos os grupos GT e GS, uma vez que foram treinados para manejar um instrumento de triagem (AUDIT) e Intervenção Breve (IB), a fim de atuarem na prevenção: "De rotina, assim, não, só daqueles que já têm uma história, mesmo, ou que eu já desconfiei do uso, aí sim, eu faço uma abordagem e converso com ele." (GS2).

Observamos a não incorporação da temática álcool e drogas numa perspectiva mais ampla, a da Saúde da Família, pois se sugere que a prevenção ao uso de risco de álcool e outras drogas deve ser ensinado junto com outros fatores de risco em geral, no contexto da saúde. As intervenções ainda são normalizadoras e estão dentro da ótica reducionista da biomedicina, sem valorização das trocas interpessoais (Alves, 2005). “(...) o que eu faço é expor os prejuizos, por exemplo, no caso dessa gestante eu vou expor o risco do aborto, do parto prematuro(...)" (GNT3). “(...) abordo mostrando para ele os riscos, os malefícios, os prejuízos que a doença traz para ele(...)" (GT2).

As ações das entrevistadas estão contradição com o modelo proposto pela OMS e pelo Ministério da Saúde (estratégia de redução de danos), cujo enfoque é biopsicossocial, numa apreensão ampliada das necessidades da população, o que sugere uma abordagem dialógica, ou seja, que se vá além da transmissão de informações sobre a saúde e se dividam as responsabilidades entre os serviços e a população assistida, transformando saberes existentes (Alves, 2005; Mattos, 2009).

Por fim, constatamos no encaminhamento dos casos graves para tratamento o que dentro da temática "práticas" fecharia o ciclo. Este começaria com a triagem, passaria pela intervenção (breve) e encaminharia os casos identificados para serviços especializados (SBIRT - Screening, Brief Intervention, and Referral for Treatment, ou seja, triagem, intervenção breve e encaminhamento para tratamento) (Babor et al., 2007). Nesse processo há um entrave, pois os municípios de pequeno porte não dispõem de CAPS AD e desconhecem alternativas, e aqueles que dispõem desse serviço muitas vezes não dão conta da demanda.

“(...) falha que existe porque, para onde você vai encaminhar? (...) Tem que ser CAPS AD, a gente não tem uma referência de CAPS $\mathrm{AD}$, então aí é uma crítica construtiva que eu faço... aí a situação volta para a gente!" (GT2). “(...) minha esperança é que quando criar um CAPS maior, que aí a gente possa fazer isso... regionalizar." (GS2).

\section{Temas 3, 4 e 5: Atitudes, Crenças, Sentimentos}

Os grupos treinados (GT e GS) apresentaram uma visão mais flexível da problemática, distante de uma concepção moralista, corroborando um estudo anterior de Ronzani et al. (2009), o qual já havia confirmado esta influência da capacitação neste grupo GT, que fora submetido naquele período a uma avaliação qualiquantitativa, com a utilização da Escala de Moralização do Uso de Álcool (EMUA) na précapacitação e avaliação de seguimento de seis meses.

“(...) problema mesmo de saúde pública, de falta de controle do governo." (GS2) "Porque é difícil eu falar assim "não fume mais, não cheire mais", porque, se ele for viciado? Porque se ele não tiver nada, uma ação recreativa, uma atividade, não tem lazer." (GT2)

Em contraposição à fala do grupo não treinado, assim se expressa o GNT3:

"Porque a gente pensa assim: "Ah, uso de álcool e droga é uma coisa ilícita". Se é ilícita, a pessoa faz isso escondido e tal... então eu vou estar assim (...) cutucando ali no segredo da pessoa (...). Então é uma invasão." (GNT3).

Cinco das sete entrevistadas se declararam despreparadas para abordar esse problema no cotidiano da assistência. Isto reforça a posição de estudos segundo os quais o sentimento de baixa autoeficácia pode ser uma barreira importante à adesão a um protocolo (guideline), reforçando a ideia de que conhecimentos e atitudes são necessários, mas não suficientes para que haja essa adesão (BoschCapblanch \& Garner, 2008; Rassool \& Rawaf, 2008). “(...) eu acredito que a dificuldade é assim, é você saber o que falar, embora ele não vá parar de beber com o que você diga." (GT2) "O desconhecimento gera medo, o desconhecimento de como abordar, como conduzir, isso aí gera essa insegurança... Que vira um medo (...) não seria bem um medo, mas a insegurança de estar abordando pela falta de capacitação.” (GS2)

\section{Tema 6: Conhecimentos sobre Intervenções e tratamentos}

Por fim, o tema "Conhecimentos específicos sobre tratamentos para álcool e outras drogas" evidenciou a expectativa dos grupos quanto ao trabalho multiprofissional, o que mantém a temática álcool e outras drogas fora do contexto da APS, ou 
seja, na antiga lógica do encaminhamento, referência e contrarreferência. A pergunta que lhes foi feita - vale lembrar - foi: "Você acredita que os usuários de álcool e outras drogas se beneficiem de algum tipo de abordagem ou tratamento específico? Você tem esta experiência de trabalho?" Ou seja, a pergunta as incluía e não privilegiava dependentes de álcool ou drogas. Ainda não vemos sinais de Rede de Atenção, com a responsabilidade dividida entre todos os envolvidos no acolhimento e/ou nas ações de cuidado continuado, tampouco a inserção do tema na lógica da prevenção e promoção de saúde. De cuidados de uma equipe multiprofissional precisam os casos graves (Cruz \& Ferreira, 2006).

\section{CONSIDERAÇÕES FINAIS}

Os procedimentos de capacitação demonstraram ter uma participação importante na mudança da visão do problema como pertencente à esfera privada e moral para uma visão menos reducionista, que considera outros fatores no desencadeamento do problema, mas não foram capazes de introduzir o tema na rotina da assistência, com a adoção de práticas baseadas em evidências. Isto se deveu a razões aqui já discutidas, entre as quais se destacou a falta de espaço para discussão permanente das deficiências, adaptações e troca de experiências, deixando o profissional vulnerável a sentimentos negativos como falta de preparo, desorientação e medo.

Os grupos treinados (GT e GS) demonstraram não utilizar em suas rotinas nenhum tipo de instrumento para rastreamento de problemas com álcool e outras drogas, o que compromete a identificação dos casos que mereçam uma intervenção. Esta, quando acontece, ainda é direcionada aos casos graves e desprovida de critérios que permitam instrumentalizar o paciente com recursos para mudança de hábitos e uma avaliação posterior. Esta prática não difere da do grupo não treinado (GNT).

Este estudo nos possibilitou chegar a algumas conclusões que talvez possam contribuir para estudos futuros que também visem identificar barreiras ao processo de educação de profissionais de saúde no tocante a álcool e outras drogas. Embora os problemas relacionados a estes sejam prevalentes em vários países e considerados um grave problema de saúde pública, muito pouco se tem feito no sentido de ampliar o acesso dos profissionais de saúde às informações relativas às habilidades específicas para se detectar o problema precocemente e intervir de forma eficaz. Neste sentido, o exercício da reflexão sobre as práticas vividas, proporcionado muitas vezes pela educação permanente, é que podem produzir o contato com o desconforto e, depois, a disposição para produzir alternativas de práticas e de conceitos para enfrentar o desafio de produzir transformações.

Os resultados encontrados e discutidos acima sugerem a necessidade de repensar o planejamento das práticas educativas em saúde, especialmente em álcool e outras drogas, uma vez que estas ações se darão dentro de um contexto, com suas crenças, valores, práticas e modelos assistenciais vigentes.

Constatamos uma prevalência do modelo curativo dicotômico, com práticas higienistas ainda hegemônicas e distantes de uma visão mais abrangente das necessidades de saúde do sujeito.

Diante de tudo isso, avaliar o que já vem sendo feito no país em termos de capacitação dos profissionais de saúde da APS para detecção e intervenção dos problemas relacionados ao álcool e outras drogas torna-se premente, já que recursos são investidos na expectativa de mudar um quadro de omissão em relação ao diagnóstico e tratamento de casos dessa ordem.

\section{AGRADECIMENTOS}

À Secretaria Nacional de Políticas sobre Drogas (SENAD) por ter disponibilizado a lista com os nomes dos alunos do curso SUPERA de 2006/2007.

Ao $\mathrm{CNPq}$, que financiou parte desta pesquisa no que tange ao projeto "Disseminação de Práticas de Prevenção ao Uso de Risco de Álcool em Serviços de APS da Zona da Mata Mineira", Edital MCT/CNPq/MS-SCTIE-DECIT 23/2006 - Estudo de Gestão em Saúde, processo 408753/2006-4.

\section{REFERÊNCIAS}

Alves, V. S. (2005). Um modelo de educação em saúde para o Programa Saúde da Família: pela integralidade da atenção e reorientação do modelo assistencial. Interface: comunicação, saúde, educação, 9(16), 39-52.

Babor, T. F., \& Higgins-Biddle, J. C. (2001). Brief Intervention for Hazardous and Harmful Drinking: a manual for use in primary care. Geneva: WHO.

Babor, T. F., Higgins-Biddle, J., Dauser, D., Higgins, P., \& Burleson, J. A. (2005). Alcohol screening and brief intervention in primary care settings: implementation models and predictors. Journal of studies on alcohol, 66(3), 361-368.

Babor, T. F., McRee, B. G., Kassebaum, P. A., Grimaldi, P. L., Ahmed, K., \& Bray, J. (2007). Screening, Brief Intervention, and Referral to Treatment (SBIRT): 
toward a public health approach to the management of substance abuse. Substance abuse, 28(3), 7-30.

Bardin, L. (1977). Análise de conteúdo (L. A. Reto \& A. Pinheiro, trad.). Lisboa: Edições 70.

Bosch-Capblanch, X., \& Garner, P. (2008). Primary health care supervision in developing countries. Tropical medicine \& international health, 13(3), 369-383.

Cabana, M. D., Rand, C. S., Powe, N. R., Wu, A. W., Wilson, M. H., Abboud, P. A., \& Rubin, H. R. (1999). Why don't physicians follow clinical practice guidelines? A framework for improvement. The journal of the American Medical Association, 282(15), 14581465 .

Camargo Júnior, K. R., Saraiva, E. M., BustamanteTeixeira, M. T., Mascarenhas, M. T. M., Mauad, N. M., \& Franco, T. B., et al. (2008). Avaliação da atenção básica pela ótica político-institucional e da organização da atenção com ênfase na integralidade. Cadernos de saúde pública, 24(Suppl. 1), 58-68.

Cantillon, P., \& Jones, R. (1999). Does continuing medical education in general practice make a difference? British medical journal, 318(7193), 1276-1279.

Cavazza, N. (2008). Psicologia das atitudes e opiniões (A. A. Machado, trad.). São Paulo: Loyola.

Ceccim, R. B. (2007). Um sentido muito próximo ao que propõe a educação permanente em saúde. Interface: comunicação, saúde, educação, 11(22), 343-363.

Cruz, M. S., \& Ferreira, S. M. B. (2006). O vínculo necessário entre a saúde mental e o PSF na construção da rede de atenção integral aos problemas relacionados ao uso de álcool e outras drogas. In: Secretaria Nacional Antidrogas. As redes comunitária e de saúde no atendimento aos usuários e dependentes de substâncias psicoativas, (pp. 42-48. (Sistema para detecção do uso abusivo e dependência de substâncias psicoativas: Encaminhamento, Intervenção Breve, Reinserção Social e Acompanhamento, Módulo 6). Brasília, DF: a Secretaria.

Garcia, R. M., \& Baptista, R. (2007). Educação à distância para a qualificação dos profissionais do SUS: perspectivas e desafios. Revista baiana de saúde pública, 31(Suppl. 1), 70-78.

Humeniuk, R., \& Poznyak, V. (2004). Intervenção breve para o abuso de substâncias: guia para uso na Atenção Primária à Saúde (T. M. Ronzani, trad.). São Paulo: OMS.

Kaner, E. F., Dickinson, H. O., Beyer, F., Pienaar, E., Schlesinger, C., \& Campbell, F. et al. (2009). Effectiveness of brief alcohol interventions in primary care settings: a systematic review. Drug and alcohol review, 28(3), 301-323.

Laranjeira, R., \& Romano, M. (2004). Consenso brasileiro sobre políticas públicas do álcool. Revista brasileira de psiquiatria, 26(Suppl. 1), 68-77.

Laranjeira, R., Pinsky, I., Zaleski, M., \& Caetano, R. (Orgs.). (2007). I Levantamento nacional sobre os padrões de consumo de álcool na população de álcool na população brasileira. Brasília, DF: Secretaria Nacional Antidrogas.
Luis, M. A. V., Mendes, I. A. C., Carvalho, E. C., Mamede, M. V., Wright, M. G. M., \& Chisman, A. McG. (2004). Experiencia de cooperación entre universidad y organización internacional para capacitar enfermeros docentes de La America Latina para La investigación del fenómeno de las drogas. Revista Latino-Americana de enfermagem, 12 (n. esp.), 307-315.

Mattos, R. A. (2009). Os sentidos da integralidade: algumas reflexões acerca de valores que merecem ser defendidos. In: R. Pinheiro \& R. A. Mattos (Orgs.), Os sentidos da integralidade na atenção e no cuidado à saúde (6a ed., pp.39-64). Rio de Janeiro: IMS/ABRASCO.

Minayo, M. C. de S. (2006). O desafio do conhecimento: pesquisa qualitativa em saúde (9a ed.). São Paulo: Hucitec.

Ministério da Saúde. Secretaria Executiva. Secretaria de Atenção a Saúde. Coordenação Nacional de DST/AIDS (2004). A política do Ministério da Saúde para atenção integral a usuários de álcool e outras drogas. (2a ed.). Brasília, DF: O Ministério.

Oliveira, M. A. N. (2007). Educação à distância como estratégia para a educação permanente em saúde: possibilidades e desafios. Revista brasileira de enfermagem, 60(5), 585-589.

Portaria n. 305 (2002, 30 de abril). Aprova as Normas de Funcionamento e Cadastramento de Centros de Atenção Psicossocial para Atendimento de Pacientes com Transtornos Causados pelo Uso Prejudicial e/ou Dependência de Álcool e Outras Drogas. Recuperado em 15 de julho de 2008, de http://dtr2001.saude.gov.br/sas/PORTARIAS/PORT200 2/PT-305.htm.

Portaria n. 816 (2002, 30 de abril). Instituir, no âmbito do Sistema Único de Saúde, o Programa Nacional de Atenção Comunitária Integrada a Usuários de Álcool e Outras Drogas. Recuperado em 15 de julho de 2008, de http://bvsms.saude.gov.br/bvs/saudelegis/gm/2002/prt0 816_30_04_2002.html.

Rassool, G. K., \& Rawaf, S. (2008). Educational intervention of undergraduate nursing students' confidence skills with alcohol and drug misusers. Nurse education today, 28(3), 284-292.

Ronzani, T. M. (2005). Avaliação de um processo de implementação de estratégias de prevenção ao uso excessivo de álcool em serviços de atenção primária à saúde: entre o ideal e o possível. Tese de Doutorado, Escola Paulista de Medicina, Universidade Federal de São Paulo, São Paulo.

Ronzani, T. M., Mota, D. C. B., \& Souza, I. C. W. (2009). Prevenção do uso de álcool na atenção primária em municípios do estado de Minas Gerais. Revista de saúde pública, 43(Suppl. 1), 51-61.

Saunders, J. B., Aasland, O. G., Babor, T. F., de la Fuente, J. R., \& Grant, M. (1993). Development of the alcohol use disorders identification test (AUDIT): WHO collaborative project on early detection of persons with harmful alcohol consumption II. Addiction, 88(6), 791804. 
Solberg, L. I., Maciosek, M. V., \& Edwards, N. M. (2008). Primary Care Intervention to Reduce Alcohol Misuse: Ranking Its Health Impact and Cost Effectiveness. American journal of preventive medicine, 34(2), 143152.

WHO Assist Working Group. (2002). The Alcohol, Recebido em 20/06/2010 Smoking and Substance Involvement Screening Test Aceito em 05/06/2012 (ASSIST): development, reliability and feasibility. Addiction, 97(9), 1183-1194.

\section{Endereço para correspondência:} Isabel Cristina Weiss de Souza. Rua Santo Antônio, 1.500/512- Centro, CEP 36016-211, Juiz de Fora-MG, Brasil.E-mail: isabel.weiss8@gmail.com. 